\title{
Autologous chondrocyte implantation versus ACI using 3D-bioresorbable graft for the treatment of large full-thickness cartilage lesions of the knee
}

Erggelet, C ; Kreuz, P C ; Mrosek, E H ; Schagemann, J C ; Lahm, A ; Ducommun, P P ; Ossendorf, C

\begin{abstract}
BACKGROUND: In autologous chondrocyte implantation (ACI), the periosteum patch which is sutured over the cartilage defect has been identified as a major source of complications such as periosteal hypertrophy. In the present retrospective study, we compared midterm results of first-generation ACI with a periosteal patch to second generation ACI using a biodegradable collagen fleece (BioSeed-C) in 82 patients suffering from chronic posttraumatic and degenerative cartilage lesions of the knee. METHODS: Clinical outcome was assessed in 42 patients of group 1 and in 40 patients of group 2 before implantation of the autologous chondrocytes and at a minimum follow-up of 2 years using the ICRS score, the modified Cincinnati score and the Lysholm score. RESULTS: Although patients treated with BioSeed-C had more previous surgical procedures on their respective knees, highly significant improvements $(\mathrm{P}<0.001)$ were assessed in both groups at comparable outcome levels: the ICRS score improved from grade D (poor) preoperatively to grade C (fair); the modified Cincinnati knee score from 3.26 to 6.4 (group 1) and 3.3 and 6.88 (group 2). Lysholm score improved from 33 to 70 points (group 1) and from 47 to 78 points (group 2 ), respectively. Revision surgery was due to symptomatic periosteal hypertrophy $(\mathrm{n}=4)$, graft failure $(\mathrm{n}=3)$, plica syndrome $(\mathrm{n}=2)$ synovectomy $(\mathrm{n}=1)$ (group 1$)$; and graft failure $(\mathrm{n}=2)$, debridement $(\mathrm{n}=1)$, synovectomy $(\mathrm{n}=2)$ (group 2). CONCLUSION: These results suggest that BioSeed-C is an equally effective treatment option for focal degenerative chondral lesions of the knee in this challenging and complex patient profile.
\end{abstract}

DOI: https://doi.org/10.1007/s00402-009-0957-y

Posted at the Zurich Open Repository and Archive, University of Zurich

ZORA URL: https://doi.org/10.5167/uzh-28044

Journal Article

Published Version

Originally published at:

Erggelet, C; Kreuz, P C; Mrosek, E H; Schagemann, J C; Lahm, A; Ducommun, P P; Ossendorf, C (2010). Autologous chondrocyte implantation versus ACI using 3D-bioresorbable graft for the treatment of large full-thickness cartilage lesions of the knee. Archives of Orthopaedic and Trauma Surgery, 130(8):957-964. DOI: https://doi.org/10.1007/s00402-009-0957-y 


\title{
Autologous chondrocyte implantation versus ACI using 3D-bioresorbable graft for the treatment of large full-thickness cartilage lesions of the knee
}

\author{
Christoph Erggelet • Peter C. Kreuz • Eike H. Mrosek • \\ Jan C. Schagemann • Andreas Lahm • \\ Pascal P. Ducommun · Christian Ossendorf
}

Received: 17 February 2009 / Published online: 27 August 2009

(C) Springer-Verlag 2009

\begin{abstract}
Background In autologous chondrocyte implantation (ACI), the periosteum patch which is sutured over the cartilage defect has been identified as a major source of complications such as periosteal hypertrophy. In the present retrospective study, we compared midterm results of firstgeneration ACI with a periosteal patch to second generation ACI using a biodegradable collagen fleece (BioSeed-C) in 82 patients suffering from chronic posttraumatic and degenerative cartilage lesions of the knee.

Methods Clinical outcome was assessed in 42 patients of group 1 and in 40 patients of group 2 before implantation of

C. Erggelet · P. C. Kreuz

Department of Orthopaedics and Trauma Surgery,

University of Freiburg, Hugstetter Strasse 49,

79095 Freiburg, Germany

E. H. Mrosek

Department of Orthopaedic Surgery, Kantonsspital St. Gallen,

Rohrschacher Strasse 95, 9007 St. Gallen, Switzerland

J. C. Schagemann

Department of Orthopaedics, University of Luebeck,

Ratzeburger Allee 160, 23538 Luebeck, Germany

A. Lahm

Section of Orthopaedic Research and Cell Biology,

Department of Orthopaedics and Orthopaedic Surgery,

University of Greifswald, F.-Sauerbruch-Strasse,

17475 Greifswald, Germany

P. P. Ducommun

Department of Surgery, Division of Plastic,

Reconstructive \& Aesthetic Surgery, Balgrist University Hospital,

University of Zurich, Forchstrasse 340, 8008 Zurich, Switzerland

C. Ossendorf $(\bowtie)$

Department of Surgery, Division of Trauma Surgery,

University of Zurich, Raemistrasse 100, 8091 Zurich, Switzerland

e-mail: c.ossendorf@gmx.net
\end{abstract}

the autologous chondrocytes and at a minimum follow-up of 2 years using the ICRS score, the modified Cincinnati score and the Lysholm score.

Results Although patients treated with BioSeed-C had more previous surgical procedures on their respective knees, highly significant improvements $(P<0.001)$ were assessed in both groups at comparable outcome levels: the ICRS score improved from grade D (poor) preoperatively to grade $\mathrm{C}$ (fair); the modified Cincinnati knee score from 3.26 to 6.4 (group 1) and 3.3 and 6.88 (group 2). Lysholm score improved from 33 to 70 points (group 1) and from 47 to 78 points (group 2), respectively. Revision surgery was due to symptomatic periosteal hypertrophy $(n=4)$, graft failure $(n=3)$, plica syndrome $(n=2)$ synovectomy $(n=1)$ (group 1); and graft failure $(n=2)$, debridement $(n=1)$, synovectomy $(n=2)$ (group 2$)$.

Conclusion These results suggest that BioSeed-C is an equally effective treatment option for focal degenerative chondral lesions of the knee in this challenging and complex patient profile.

Keywords Knee $\cdot$ Cartilage $\cdot$ Biosurgery $\cdot$ Trauma . Degenerative defects

\section{Introduction}

The treatment of cartilage defects represents a common, complex and multifaceted task for orthopaedic surgeons; particularly, in young patients suffering from large cartilage defects, there are only limited conservative and surgical treatment options. Thus, several efforts to restore articular cartilage were undertaken $[4,11,14,20,23$, 38]. However, these cartilage repair techniques provide only limited durability of the repair tissue [29] or 
suitable for small defects only [6]. In contrast, autologous chondrocyte implantation (ACI) introduced in 1994, lacks these disadvantages, but provides hyalinelike cartilage [4, 35-37].

Autologous chondrocyte implantation has been recommended as the treatment of choice for symptomatic cartilage defects larger than $2 \mathrm{~cm} \mathrm{[2]} \mathrm{and} \mathrm{for} \mathrm{failed} \mathrm{cartilage}$ repair procedures and more than 10,000 patients worldwide have been treated with ACI already [26]. Encouraging short to midterm results of ACI were reported from case series $[7,9,24,25,33,39]$, while randomised clinical trials could not clearly demonstrate superiority of ACI compared with either microfracture or osteochondral cylinder transplantation $[3,12,13,17,18]$. First-generation ACI involves harvesting a periosteal flap from the tibia that later has to be fixed to the surrounding cartilage requiring a contained cartilage lesion. Application in some regions of the knee may thus be delicate or even impossible. Notably, the periosteal flap covering the defect has been identified as a potential source of complications resulting in reoperations in up to $25 \%$ of the patients $[19,24,27,28,37]$. To eliminate this significant disadvantage, to prevent chondrocytes from dedifferentiation, to improve adhesion and to facilitate handling, ACI has been refined through the development of bioresorbable matrices (e.g., MACI, BioSeed-C). With these grafts, it is no longer necessary to have a stable cartilage rim surrounding the defect and non-contained lesions can be addressed as well as contained lesions. Addressing defects on the medial or lateral femoral condyle that are smaller than $2 \mathrm{~cm} \mathrm{[2],} \mathrm{BioSeed-C} \mathrm{can} \mathrm{be} \mathrm{implanted} \mathrm{arthro-}$ scopically [8].

BioSeed-C is now frequently applied to a significant patient population. Theoretically, the advantages of this technique would make it a better choice for the treatment of full-thickness cartilage lesions of the knee outclassing standard periosteum ACI. However, no study has compared BioSeed-C to the standard method of periosteum ACI yet. Therefore, orthopaedic surgeons cannot be sure of whether they deprive their patients of a potentially better articular cartilage repair if keeping at periosteum ACI. To determine which of the two techniques should be chosen to ensure best treatment results, to optimise the outcome and the benefit for the patients suffering from full-thickness cartilage lesions of the knee and to assess typical complications of both techniques, we performed a retrospective study to evaluate and to compare the clinical outcomes of standard ACI to ACI using a biodegradable polymer-fleece.

\section{Materials and methods}

In this clinical non-randomised retrospective study, 82 patients (42 women, 40 men) meeting the following criteria were included: (1) clinical and radiographic [16] focal full-thickness cartilage lesions of the knee on the medial or lateral femoral condyle, patella or trochlea; (2) lesion grades III-IV in the Outerbridge classification [34]; (3) symptom-like pain or dysfunction of the knee joint and (4) given patient's oral consent following adequate information about mode of data acquisition, processing, analysis, interpretation and publication, according to regional guidelines.

Patients fulfilling at least one of the following criteria were not included in this study: (1) severe osteoarthritis; (2) diffuse tricompartmental degeneration; (3) multiple small lesions $<1 \mathrm{~cm}$ [2]; (4) bilateral chondrocyte implantation; (5) age over 65 years; (6) presence of active infection; (7) injuries or afflictions of regions other than the knee; (8) gravity; (9) neoplasms; (10) radicular pain; (11) paralysis; (12) psychiatric diseases and (13) fibromyalgia.

Between March 1997 and October 2004, 82 patients were treated with ACI for full-thickness cartilage lesions of the knee in one hospital. They formed two groups: group 1 was treated with standard periosteum ACI and group 2 with ACI using a biodegradable fleece (BioSeed-C). There were 29 men and 13 women in group 1 and 22 men and 18 women in group 2. Mean defect size was $6.38 \mathrm{~cm}^{2}(2-17.5)$ in group 1 and $4.6 \mathrm{~cm}^{2}(2-15)$ in group $2(P<0.001)$. Average age was 34 (16-53) and 36 years (17-64), respectively. In both groups, defects were mainly situated on the medial femoral condyle (group 1: $n=29$, group 2: $n=27$ ). Mean follow-up of the periosteum ACI group was 36 months (24-63), of the BioSeed-C group 24 months (24 months). All but three patients of group 1 had at least one previous surgical procedure on the knee, with a mean of $1.94(0-4)$. In group 2, all patients had previous surgical procedures of the knee with a mean of 3.35 (1-11), including meniscectomies (group 1: $n=20$, group 2: $n=20$ ), reconstructions of the anterior cruciate ligament (group 1: $n=10$, group 2: $n=12$ ) and cartilage repair procedures like abrasion arthroplasty (group 1: $n=10$, group 2: $n=0$ ), microfractures and drillings (group 1: $n=17$, group 2: $n=13$ ), respectively. In patients with the disruption of the anterior cruciate ligament, ACL reconstruction is required and was performed together with ACI; so was done with malalignment. More detailed information about the study cohorts is given in Table 1.

ICRS score [15], Lysholm score [21], Cincinnati score $[31,32]$ and the modified Cincinnati knee score [30] were used for evaluation of outcome. Data acquisition was performed by an independent investigator. Clinical evaluation and scoring were done preoperatively and at follow-up. To calculate levels of significance, the paired and unpaired Wilcoxon-rank sum test was done with SPSS for Windows Version 11.0 (SPSS, Chicago, IL, USA). 


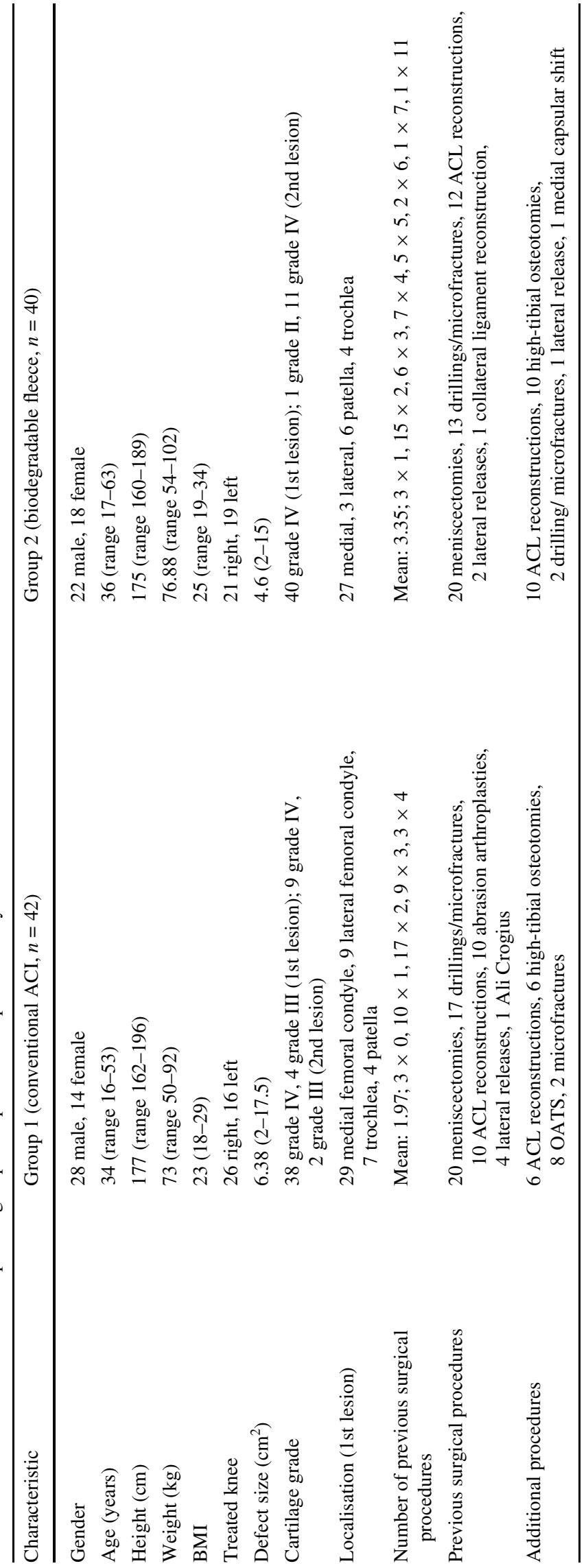




\section{Surgical technique}

For both procedures, periosteum ACI and BioSeed-C, the cartilage defect was assessed arthroscopically for definite indication to ACI. Approximately, $250 \mathrm{mg}$ of articular cartilage was taken as a biopsy from a lesser or non-weight bearing region of the knee as the linea terminalis or the intercondylar notch. The biopsy was placed in transport container provided by the commercial cell culturing company and sent to the respective company's cell culturing facility (Genzyme Biosurgery, Cambridge, MA, USA; Biotissue Technologies, Freiburg, Germany). There, chondrocytes were expanded in vitro. For conventional ACI, these chondrocytes were brought into a suspension at for later injection. For BioSeed-C, the chondrocytes were rearranged three dimensionally in fibrin and a polymer-based scaffold of polyglycolic/polylactic acid (polyglactin, vicryl) and polydioxanone.

The implantation of the cultured autologous chondrocytes was performed at a date according to the laboratory guidelines. Under general or local anaesthesia and antibiotic prophylaxis, a medial or lateral arthrotomy was performed preparing the cartilage defect in a tourniquet-controlled bloodless field. The cartilage lesion was carefully debrided back to healthy cartilage building a stable rim.

For periosteum ACI, a template was fitted to defect size and periosteum was harvested from the lateral aspect of the tibia using this template. This periosteal flap was fitted to defect size and sutured into the defect cambium layer down (Vicryl 6-0) leaving a gap for the injection of the cultured chondrocytes (Fig. 1 b, arrowhead). The rim was sealed with fibrin glue. The chondrocyte suspension was injected under the periosteal flap and the flap was finally secured and sealed with fibrin glue and a final suture.

BioSeed-C is a resorbable composite material consisting of polyglaction 910 and poly-p-dioxanone collagen fleece seeded with autologous chondrocytes in a three-dimensional matrix. It contains $20 \times 10^{6}$ cells $/ \mathrm{cm}^{3}$ fixed with approximately $5-7 \mathrm{mg}$ fibrin adhesive and will be resorbed after 2-3 months. For the BioSeed-C procedure, the defect was debrided to a rectangular shape down to the subchondral bone using a sharp curette (Fig. 2d). A template was taken and the fleece fitted to size. Using a resorbable thread, the graft (Fig. 2a) was armed with a double-knot loop at each corner (Vicryl 2-0). One threefold knot approximately $1 \mathrm{~cm}$ from the edge secures the sling (Fig. 2b). An additional knot approximately $1 \mathrm{~cm}$ out moors the sling and serves as a pulley. Using k-wires $(1.7 \mathrm{~mm}, 35 \mathrm{~cm}$ long with eye), four drill holes on the corners of the defect were made to fix the implant transosseously (Fig. 2c). Firm action on the pulleys guided the knots into the transosseous drill holes at the corners of the defect (Fig. 2d). The pulley slings were cut close to their dermal exit (Fig. 2e).

For both procedures, arthrotomy was closed under careful haemostasis. For postoperative management, continuous passive motion machines were used starting the first postoperative day. Range of motion initially was limited to $40^{\circ}$ of flexion and was increased in stages of $5^{\circ}-10^{\circ}$ per day limited to $90^{\circ}$ of flexion. The operated knee was unloaded for 6 weeks with approximately $15 \%$ of the body weight. Physiotherapy was prescribed individually. Starting the seventh postoperative week, load was increased gradually and specific isometric strengthening exercises were performed. Light exercise such as walking or cycling was
Fig. 1 Periosteum ACI: the cartilage defect was debrided back to healthy cartilage (left). A periost patch harvested from the tibia was sutured into the defect cambium layer down. On the upper left side, one suture was skipped (arrow) which will be done later to allow injection of the autologous chondrocyte suspension (right)
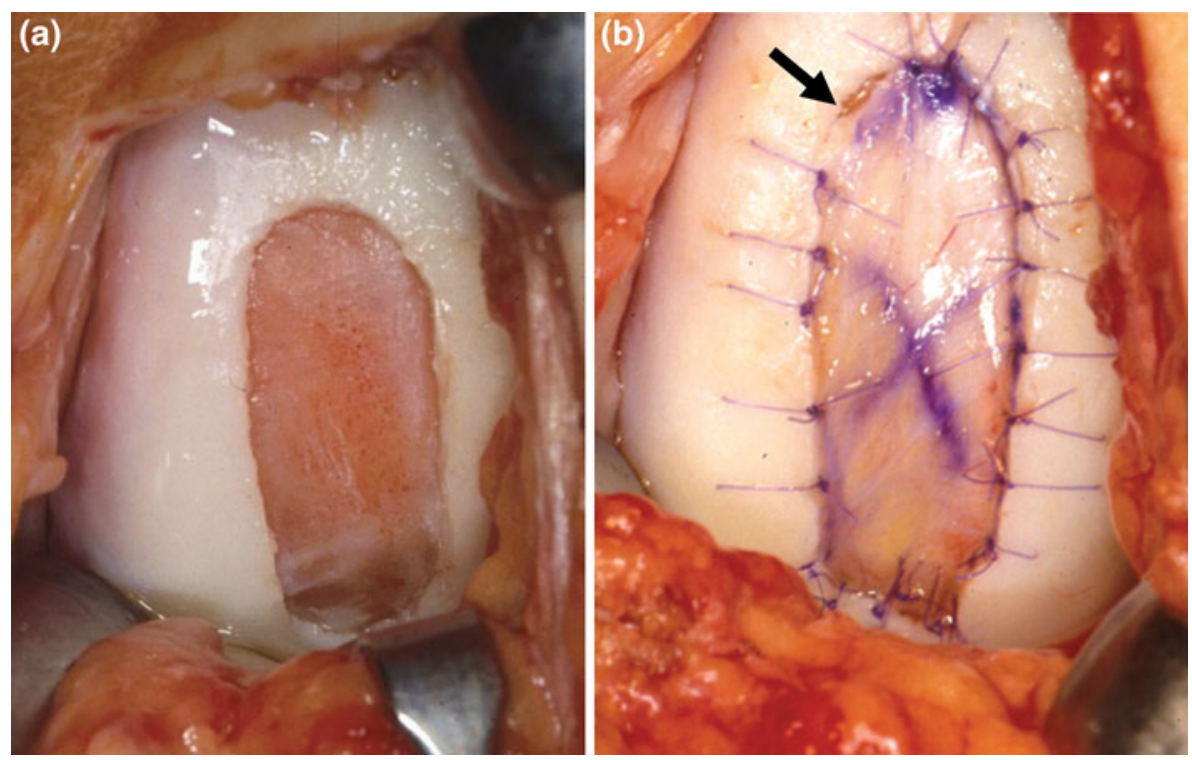
Fig. 2 BioSeed-C is delivered as a $20 \times 30 \times 2 \mathrm{~mm}$ polymerfleece seeded with $2 \times 10^{6}$ autologous chondrocytes (a). The graft is armed with vicryl sutures at every corner which serve as a pulley (b) and are fixed transosseously using 1.7-mm-k-wires in an insideout technique (c); that way, the graft is smoothly fitted into the defect (d) and securely fixed in a press-fit technique (e)
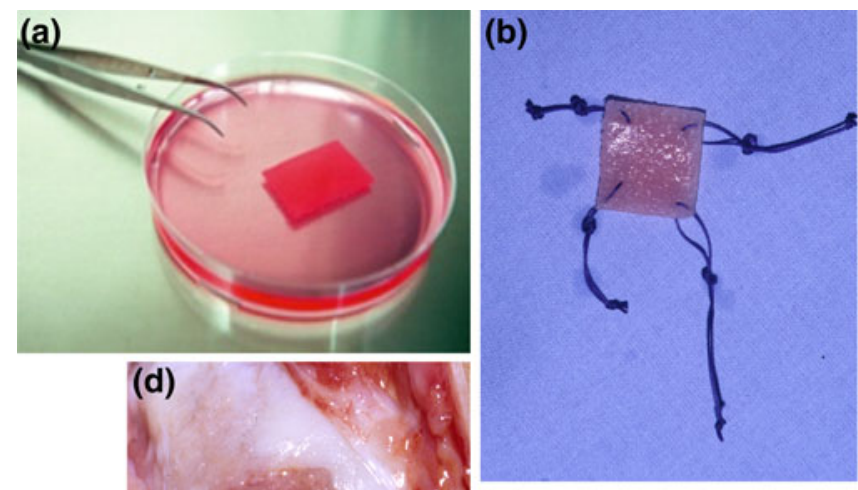

(c)
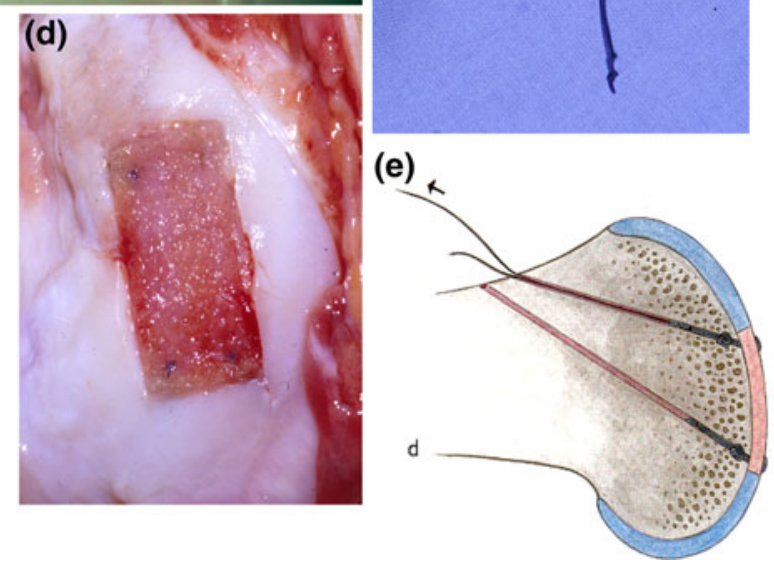

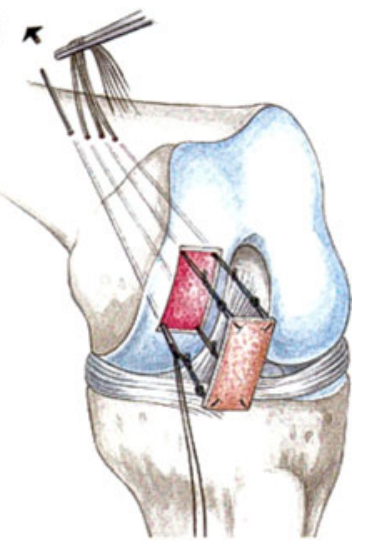

allowed after the sixth postoperative month and return to contact sports was not recommended before 12 months postoperatively.

\section{Results}

Intraoperatively, no loosening, ablation or derangement of either transplant occurred. No knee joint infection and no allergic reaction occurred in either group. Postoperatively, neither an extension lag nor a flexion deficit could be observed. One patient of the BioSeed-C group had a moderate effusion at follow-up, but not other problems and did well later on. The rate of follow-up in the periosteum group was $100 \%$. In the BioSeed-C group, the grafts of one patient were removed in a peripheral institution due to soft regeneration tissue after 9 months. This patient was registered as a treatment failure. Another patient developed paraplegia during follow-up and, therefore, was excluded from the study. One patient was lost to follow-up due to unknown address.

At follow-up, mean scores significantly increased in both groups compared with baseline: in the periosteal group, the ICRS score improved from grade D (poor) preoperatively to grade $\mathrm{C}$ (fair) post-op $(P<0.0001)$ with a range from grade A (excellent) to grade D (poor) (Fig. 3a). The modified Cincinnati knee score improved from 3.26 to 6.44 (patient) $(P<0.0001)$ and from 3.36 to 6.88 (physician) $(P<0.0001)$ (Fig. 3 b, c). Mean Lysholm score improved from 33 to 70 points $(P<0.0001)$ (Fig. 3d).

In BioSeed-C group, like in the periosteum group, the ICRS score improved from grades $\mathrm{D}$ to $\mathrm{C}$ as well
$(P<0.0001)$ (Fig. 3a). The modified Cincinnati knee score increased from 3.3 to 6.4 (patient) $(P<0.0001)$ and from 4.9 to 6.5 (physician) $(P<0.0001)$ (Fig. 3b, c). Mean Lysholm score enhanced from 47 to 78 points $(P<0.0001)$ (Fig. 3d). There was no significant difference between groups 1 and 2 in the ICRS score rating $(P=1)$ and in the Lysholm score $(P=0.065)$. In the overall rating of the modified Cincinnati knee score (patient), the difference between groups was not statistically significant $(P=0.39)$. However, the physician rating was significantly better in the periosteum group $(P=0.033)$.

In the periosteum group, 10 patients required revision surgery, due to symptomatic periosteal hypertrophy $(n=4)$ or graft failure $(n=3)$, plica syndrome $(n=2)$ and synovectomy $(n=1)$.

In the BioSeed-C group, reoperations were necessary and regarded as directly related to ACI in five patients. One patient underwent graft removal in a peripheral institution and was thus registered as a treatment failure. Two patients had a synovectomy and one patient had a debridement and one a total knee replacement, respectively.

\section{Discussion}

The aim of the present study was to compare the clinical outcome of standard periosteum ACI to BioSeed-C (ACI using a biodegradable fleece) in patients with focal degenerative cartilage defects of the knee. Patients of both treatment groups showed highly significant increases in the respective scores used for outcome measurement 
Fig. 3 Clinical outcome after 2 years as measured by the ICRS, modified Cincinnati knee score and the Lysholm score. All scores showed highly significant $(P<0.0001)$ improvements at follow-up. The ICRS score improved from grade IV at baseline to grade III at follow-up (a). The modified Cincinnati knee score as evaluated by patient (b) and physician (c) showed highly significant improvements in the evaluated follow-up period. The Lysholm score improved highly significant as well (d)
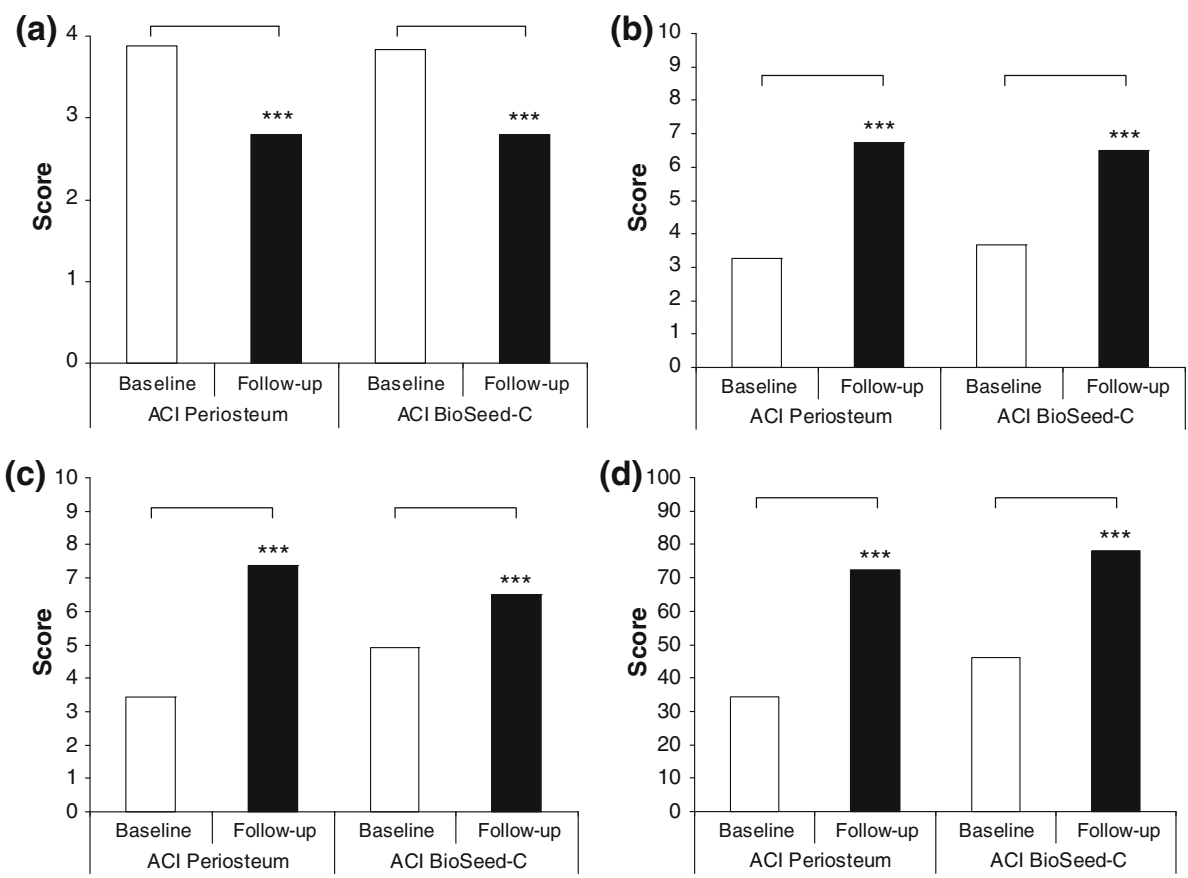

demonstrating major improvements in activities of daily living, ability to work and in sports. Patients treated with periosteal ACI required twice as many reoperations as patients treated with BioSeed-C. In the ICRS score, the patient rating of the modified Cincinnati knee score and the Lysholm score, the outcome was comparable between the periosteum and the BioSeed-C group. However, in the objective and strict ICRS score and in the patient rating of the modified Cincinnati knee score, patients treated with periosteum ACI scored slightly, but not significantly better, whereas in the Lysholm score, it was vice versa. In contrast, in the physician's rating, BioSeed-C patients scored slightly worse than periosteum patients, because of better baseline ratings.

The patients of the two groups compared in this study differed in defect size, number of previous or concomitant surgical procedures and length of the postoperative followup period. This may influence the results. However, the impact of this potential effect is unknown. The group treated with conventional ACI had larger defects and longer follow-up. However, a direct comparison of the two groups may be inappropriate, as there were significantly more previous surgical procedures in the group treated with BioSeed-C.

When compared with case series, randomised studies evaluating ACI versus other cartilage repair surgeries as reported previously are favourable [3, 12, 13, 17, 18]. A recent systematic review comparing ACI and osteochondral autograft transfer with each other with one another and with traditional abrasive techniques demonstrated no clear superiority of ACI over microfracture [22] in line with the findings published by other authors [17, 18]. However, in another study, ACI showed a superior cartilage regenerate than microfracture at same clinical short-term results [39].

When comparing the two ACI methods, the present study shows that these are equally effective. Advantages of the BioSeed-C techniques comprise less morbidity, as no periosteum has to be harvested and the option of arthroscopical implantation. Defects of the femoral condyles can be addressed arthroscopically depending on lesion size and location which is associated with faster recovery after surgery and with better cosmetical results. Further advantages of BioSeed-C, are that it is easier to apply, is more stable than the periosteum cartilage transplant and triggers less second look surgeries, because there can be no periosteum hypertrophy. This may partly be due to the lacking periosteum hypertrophies frequently causing reoperations in patients treated with ACI using periosteum. ACI involves an open technique with inherent disadvantages such as adhesions and prolonged recovery. Therefore, an arthroscopical approach to ACI as published previously is desirable and could potentially reduce postoperative morbidity [8]. The authors believe that this is the first study comparing standard ACI to a 3D fleece technique (BioSeed-C), representing a non-randomised retrospective, comparative study with at least 40 patients in each group. However, the outcome of this study should be interpreted with care and statistical effects associated with small numbers of patients may be considered.

First-generation tissue engineering grafts such as periosteum ACI have been demonstrated to be an appropriate therapy for the regeneration of posttraumatic defects $[5,10]$. However, second generation cartilage tissue 
engineering grafts using various matrices were recently considered to be technically more attractive. For instance, in a series of 47 cartilage defects, similar outcomes were obtained clinically as well as histologically [1]. In a multicenter study evaluating Hyalograft $\mathrm{C}$, which consists of autologous chondrocytes embedded in a derivative of hyaluronic acid, more than $90 \%$ of patients showed improvements of the ICRS score [23]. Clinical assessment as done by the Meyers score, the Lysholm score and in the ICRS scores was improved in a prospective study investigating 5-year results of matrix associated ACI [2].

In summary, we regard the outcome of this study as a decent treatment result for this difficult and highly demanding patient profile, particularly because patients treated with BioSeed-C had the same outcome levels, e.g., in kneerelated performance and quality of life as the patients treated with periosteum ACI, although they have had more surgeries on their knees before.

Concerning its advantages in comparison to periosteum $\mathrm{ACI}$, we conclude that BioSeed-C is equally effective as periosteum ACI.

Conflict of interest statement All authors certify they have not signed any agreementwith a commercial interest related to this study which would in any way limit publicationof any and all data generated for the study or to delay publication for any reason.

\section{References}

1. Bartlett W, Skinner JA, Gooding CR et al (2005) Autologous chondrocyte implantation versus matrix-induced autologous chondrocyte implantation for osteochondral defects of the knee: a prospective, randomised study. J Bone Joint Surg 87-B:640-645

2. Behrens P, Bitter T, Kurz B et al (2006) Matrix-associated autologous chondrocyte transplantation/implantation (MACT/MACI): 5-year follow-up. Knee 13:194-202

3. Bentley G, Biant LC, Carrington RW et al (2003) A prospective, randomised comparison of autologous chondrocyte implantation versus mosaicplasty for osteochondral defects in the knee. J Bone Joint Surg 85-B:223-230

4. Brittberg M, Lindahl A, Nilsson A et al (1994) Treatment of deep cartilage defects in the knee with autologous chondrocyte transplantation. N Engl J Med 331:889-895

5. Browne JE, Anderson AF, Arciero R et al (2995) Clinical outcome of autologous chondrocyte implantation at 5 years in US subjects. Clin Orthop Relat Res 436:237-245

6. Curl WW, Krome J, Gordon ES et al (1997) Cartilage injuries: a review of 31, 516 knee arthroscopies. Arthroscopy 13:456-460

7. Erggelet C, Browne JE, Fu F et al (2000) Autologous chondrocyte transplantation for treatment of cartilage defects of the knee joint: clinical results. Zentralbl Chir 125:516-522

8. Erggelet C, Sittinger M, Lahm A (2003) The arthroscopic implantation of autologous chondrocytes for the treatment of full-thickness cartilage defects of the knee joint. Arthroscopy 19:108-110

9. Erggelet C, Steinwachs MR, Reichelt A (2000) The operative treatment of full thickness cartilage defects in the knee joint with autologous chondrocyte transplantation. Saudi Med J 21:715-721

10. Fu FH, Zurakowski D, Browne JE et al (2005) Autologous chondrocyte implantation versus debridement for treatment of full-thickness chondral defects of the knee: an observational cohort study with 3-year follow-up. Am J Sports Med 33:1658-1666

11. Hangody L, Karpati Z (1994) New possibilities in the management of severe circumscribed cartilage damage in the knee. Magy Traumatol Ortop Kezseb Plasztikai Seb 37:237-243

12. Horas U, Pelinkovic D, Herr G et al (2003) Autologous chondrocyte implantation and osteochondral cylinder transplantation in cartilage repair of the knee joint: a prospective, comparative trial. J Bone Joint Surg 85-A:185-192

13. Horas U, Schnettler R, Pelinkovic D et al (2000) Osteochondral transplantation versus autogenous chondrocyte transplantation: a prospective comparative clinical study. Chirurg 71:1090-1097

14. Hubbard MJ (1996) Articular debridement versus washout for degeneration of the medial femoral condyle: a five-year study. J Bone Joint Surg Br 78:217-219

15. International Cartilage Repair Society (1999) http://www.cartilage.org

16. Kellgren JH, Lawrence JS (1957) Radiological assessment of rheumatoid arthritis. Ann Rheum Dis 16:485-493

17. Knutsen G, Drogset JO, Engebretsen L et al (2007) A randomized trial comparing autologous chondrocyte implantation with microfracture: findings at five years. J Bone Joint Surg 89-A:2105-2112

18. Knutsen G, Engebretsen L, Ludvigsen TC et al (2004) Autologous chondrocyte implantation compared with microfracture in the knee: a randomized trial. J Bone Joint Surg Am 86-A:455-464

19. Kreuz PC, Steinwachs M, Erggelet $C$ et al (2007) Classification of graft hypertrophy after autologous chondrocyte implantation of full-thickness chondral defects in the knee. Osteoarthritis Cartilage 15:1339-1347

20. Lexer E (1908) Substitution of whole or half joints from freshly amputated extremities by free plastic operation. Surg Gynecol Obstet 6:601-608

21. Lysholm J, Gillquist J (1982) Evaluation of knee ligament surgery results with special emphasis on use of a scoring scale. Am J Sports Med 10:150-154

22. Marcacci M, Berruto M, Brocchetta D et al (2005) Articular cartilage engineering with hyalograft C: 3-year clinical results. Clin Orthop Relat Res 435:96-105

23. Matsusue Y, Yamamuro T, Hama H (1993) Arthroscopic multiple osteochondral transplantation to the chondral defect in the knee associated with anterior cruciate ligament disruption. Arthroscopy 9:318-321

24. Micheli LJ, Browne JE, Erggelet C et al (2001) Autologous chondrocyte implantation of the knee: multicenter experience and minimum 3-year follow-up. Clin J Sport Med 11:223-228

25. Minas T (2001) Autologous chondrocyte implantation for focal chondral defects of the knee. Clin Orthop Relat Res 391:349361

26. Minas T (2003) Autologous chondrocyte implantation in the arthritic knee. Orthopedics 26:945-947

27. Minas T (1999) The role of cartilage repair techniques, including chondrocyte transplantation, in focal chondral knee damage. Instr Course Lect 48:629-643

28. Minas T, Peterson L (1999) Advanced techniques in autologous chondrocyte transplantation. Clin Sports Med 18:13-44, v-vi

29. Nehrer S, Spector M, Minas T (1999) Histologic analysis of tissue after failed cartilage repair procedures. Clin Orthop Relat Res 365:149-162

30. Noyes FR, Barber-Westin SD (1997) Arthroscopic-assisted allograft anterior cruciate ligament reconstruction in patients with symptomatic arthrosis. Arthroscopy 13:24-32

31. Noyes FR, Barber SD, Mooar LA (1989) A rationale for assessing sports activity levels and limitations in knee disorders. Clin Orthop Relat Res 246:238-249

32. Noyes FR, McGinniss GH, Mooar LA (1984) Functional disability in the anterior cruciate insufficient knee syndrome: review of knee 
rating systems and projected risk factors in determining treatment. Sports Med 1:278-302

33. Ossendorf C, Kreuz PC, Steinwachs MR et al (2007) Autologous chondrocyte implantation for the treatment of large full-thickness cartilage lesions of the knee. Saudi Med J 28:1251-1256

34. Outerbridge RE (1961) The etiology of chondromalacia patellae. J Bone Joint Surg 43-B:752-757

35. Peterson L (1996) Articular cartilage injuries treated with autologous chondrocyte transplantation in the human knee. Acta Orthop Belg 62(Suppl 1):196-200

36. Peterson L, Brittberg M, Kiviranta I et al (2002) Autologous chondrocyte transplantation: biomechanics and long-term durability. Am J Sports Med 30:2-12
37. Peterson L, Minas T, Brittberg M et al (2002) Two- to 9-year outcome after autologous chondrocyte transplantation of the knee. Clin Orthop Relat Res 374:212-234

38. Steadman JR, Rodkey WG, Rodrigo JJ (2001) Microfracture: surgical technique and rehabilitation to treat chondral defects. Clin Orthop Relat Res 391:S362-S369

39. Steinwachs M, Kreuz PC (2007) Autologous chondrocyte implantation in chondral defects of the knee with a type I/III collagen membrane: a prospective study with a 3-year follow-up. Arthroscopy $23: 381-387$ 